\title{
Schrödinger Operator with a Nonlocal Potential whose Absolutely Continuous and Point Spectra Coexist
}

\author{
A. L. Figotin and L. A. Pastur \\ Institute for Low Temperature Physics and Engineering, UkrSSR Academy of Science, \\ SU-310164 Kharkov, USSR
}

\begin{abstract}
We consider the Schrödinger-like operator $H$ in which the role of a potential is played by the lattice sum of rank 1 operators $\left|v_{n}\right\rangle\left\langle v_{n}\right|$ multiplied by $g \tan \pi[(\alpha, n)+\omega], g>0, \alpha \in \mathbb{R}^{d}, n \in \mathbb{Z}^{d}, \omega \in[0,1]$. We show that if the vector $\alpha$ satisfies the Diophantine condition and the Fourier transform support of the functions $v_{n}(x)=v(x-n), x \in \mathbb{R}^{d}, n \in \mathbb{Z}^{d}$, is small then the spectrum of $H$ consists of a dense point component coinciding with $\mathbb{R}$ and an absolutely continuous component coinciding with $[\varrho, \infty)$, where $\varrho$ is the radius of the mentioned support. Besides, we find the integrated density of states $N(\lambda)$ (it has a jump at $\lambda=\varrho$ ) and zero temperature a.c. conductivity $\sigma_{\lambda}(v)$, that also has a jump at $\lambda=\varrho$ and vanishes faster than any power of the external field frequency $v$ as $v \rightarrow 0$ and $\lambda \neq \varrho$.
\end{abstract}

\section{Main Results and Discussion}

The present work is devoted to the spectral analysis of an operator

$$
H=-(2 \pi)^{-2} \Delta+Q
$$

on $L_{2}\left(\mathbb{R}^{d}\right)$. Here $\Delta$ is the Laplace operator and the operator $Q$ (a pseudopotential) has the form

$$
\begin{gathered}
Q=\sum_{n \in \mathbb{Z}^{d}} t_{n}\left|v_{n}\right\rangle\left\langle v_{n}\right|, \\
\left(\left|v_{n}\right\rangle\left\langle v_{n}\right| \varphi\right)(x)=v_{n}(x)\left(v_{n}, \varphi\right), \quad v_{n}(x)=v(x-n), \\
t_{n}=\tan \pi[(\alpha, n)+\omega], \quad \omega \in[0,1) \\
\omega \neq \frac{1}{2}-(\alpha, n)(\bmod 1), \quad n \in \mathbb{Z}^{d},
\end{gathered}
$$

the vector $\alpha \in \mathbb{R}^{d}$ satisfying a Diophantine condition

$$
|(\alpha, n)-m| \geq C|n|^{-\beta}, m \in \mathbb{Z}, \quad n \in \mathbb{Z}^{d} \backslash\{0\}
$$

with positive constants $C$ and $\beta$. 
On the function $v(x)$ that appears in the definition of the pseudopotential $Q$ we shall impose

Condition $V: v(x), x \in \mathbb{R}^{d}$ is a real function whose Fourier transform

$$
\hat{v}(p)=\int_{\mathbb{R}^{d}} e^{-2 \pi i(p, x)} v(x) d x, \quad p \in \mathbb{R}^{d},
$$

is $\hat{v}(p)=a\left(p^{2}\right), p^{2}=(p, p)$, and a real function $a(\mu), \mu \geq 0$, is infinitely differentiable on $[0, \infty)$, and for some positive $\varrho$,

$$
a(\mu) \equiv 0, \mu \geq \varrho ; \quad a(\mu)>0,0 \leq \mu<\varrho .
$$

We assume that for $\varrho=\infty$ the function $a$ and all its derivatives decrease faster than any power of $\mu^{-1}$ when $\mu \rightarrow \infty$.

The factor $(2 \pi)^{-2}$ before the Laplacian in (1.1) is introduced to simplify many expressions that will appear below.

We will denote by $E(d \lambda)$ and $E_{0}(d \lambda)$ the resolutions of identity of the operators $H$ and $H_{0}=-(2 \pi)^{-2} \Delta$, respectively; we will also introduce notations: $\mathscr{H}_{-}=$ $E_{0}((-\infty, \varrho)) L_{2}\left(\mathbb{R}^{d}\right), \mathscr{H}_{+}=E_{0}((\varrho, \infty)) L_{2}\left(\mathbb{R}^{d}\right)$ and $S=S\left(\mathbb{R}^{d}\right)$ being the Schwartz space.

Theorem 1.1. The operator $H$ is essentially self-adjoint on $S$. The subspaces $\mathscr{H}_{+}$ and $\mathscr{H}$ - reduce the operator $H$, the restriction of the operator $H$ to $\mathscr{H}_{+}$coincides with the restriction of the operator $-(2 \pi)^{-2} \Delta$ to $\mathscr{H}_{+}$and for $d \lambda \subset(\varrho, \infty)$,

$$
E(d \lambda) \mathscr{H}_{+}=E_{0}(d \lambda) \mathscr{H}_{+} \cdot
$$

Thus the spectrum of the operator $H$ on the $[\varrho, \infty)$ has an absolutely continuous component.

Theorem 1.2. Let $\varrho>\frac{1}{4}$. Then all the assertions of Theorem 1.1. are valid and, besides, there exists such a positive number $\varrho_{1}$ that for almost all in the Lebesgue measure $\omega \in[0,1)$ the spectrum of the operator $H$ in the $\left(-\infty, \varrho_{1}\right)$ is pure point, dense and has multiplicity 1 , while the eigenfunctions belong to $S$.

Theorem 1.3. Let $\varrho<\frac{1}{4}$. Then all the assertions of Theorem 1.1. are valid and, besides, for almost all in the Lebesgue measure $\omega \in[0,1)$ the spectrum of the operator consists of point and absolutely continuous components, the point spectrum has multiplicity 1 , is dense in $\mathbb{R}$ and the respective eigenfunctions belong to $S$. The orthogonal projections corresponding to the point and absolutely continuous components coincide, respectively, with the projections onto $\mathscr{H}_{-}$and $\mathscr{H}_{+}$subspaces.

It is easy to see that the operator $H=H(\omega)$ investigated here, belongs to the class of metrically transitive operators $[1,2]$. In this connection, it is natural to consider the integrated density of states (IDS) $N(\lambda)$. It can be defined by the formula used in the case of Schrödinger operators with a local potential [3], viz.

$$
N(\lambda)=\int_{0}^{1} d \omega \operatorname{Sp} \chi_{\mathbb{T}} E(\lambda, \omega) \chi_{\mathbb{T}},
$$


where $\mathbb{T}$ is a unit cube and $\chi_{\mathbb{T}}$ is a multiplication by its characteristic function considered as the operator on $L_{2}\left(\mathbb{R}^{d}\right)$. The measure corresponding to a nondecreasing function $N(\lambda)$ will be denoted by $N(d \lambda)$. In [1-3] we proved the equivalence of Eq. (1.9) to the definition of the same function $N(\lambda)$ as a limit of similar functions for a system of infinitely expanding cubes for a fairly broad class of operators. Here we intend to calculate this quantity for operator (1.1)-(1.5).

Theorem 1.4. Let $\varrho<\frac{1}{4}$. Then the measure $N(d \lambda)$ is absolutely continuous, i.e. $N(d \lambda)=n(\lambda) d \lambda$ and its density (the density of states) $n(\lambda)$ is determined by the formula

$$
n(\lambda)=n_{p}(\lambda)+\chi(\lambda-\varrho) n_{0}(\lambda),
$$

where the first term corresponds to the point component of the operator $H$ spectrum and is equal to

$$
n_{p}(\lambda)=\frac{1}{\pi} \int_{p^{2} \leq \varrho} \frac{\hat{v}^{2}(p) d p}{\left(p^{2}-\lambda\right)^{2}+\hat{v}^{4}(p)}, \quad \lambda \in \mathbb{R},
$$

while the second term corresponds to the absolutely continuous component of the spectrum of $H, \chi(\lambda)$ being the characteristic function of the $[0, \infty)$, and $n_{0}(\lambda)=$ $N_{0}^{\prime}(\lambda)=\pi^{\frac{d}{2}} \Gamma^{-1}\left(\frac{d}{2}\right) \lambda^{\frac{d}{2}-1}$ being the density of states of the operator $H_{0}$. Besides if $V_{\varrho}$ is the volume of a ball of radius $\varrho$ in $\mathbb{R}^{d}$, then

$$
\int_{-\infty}^{\infty} n_{p}(\lambda) d \lambda=V_{\varrho} .
$$

Theorem 1.5. Let $\varrho>\frac{1}{4}$. Then the measure $N(d \lambda)$ is absolutely continuous on the interval $\left(-\infty, \varrho_{1}\right)$ with $\varrho_{1}$ determined in Theorem 1.2. The density $n(\lambda)$ of $N(d \lambda)$ is given by the formula

$$
n(\lambda)=\int_{\mathbb{R}^{d}}\left(\frac{\hat{v}(p)}{p^{2}-\lambda}\right)^{2}\left(w_{\lambda}(p)+1\right)^{-1} d p, \quad \lambda<\varrho_{1},
$$

with the function $w_{\lambda}(p)$ being determined by Eqs. (1.12), (1.15).

Thus, we see that the measure $N(d \lambda)$ in the $\left(-\infty, \varrho_{1}\right)$ is due to point component of the spectrum in accordance with Theorem 2 .

Theorem 1.6. Let $\varrho<\frac{1}{4}$ and $\sigma(v, \lambda)$ be the conductivity corresponding to the operator $H$ at zero temperature, external field frequency $v$ and Fermi energy $\lambda$ (rigorously defined in Sect.4). Then

$$
\sigma(v, \lambda)=\sigma_{p}(v, \lambda)+\sigma_{0}(v, \lambda),
$$

where the first term corresponds to the point while the second one to the absolutely continuous spectrum components of the operator $H$. Here $\sigma_{0}(v, \lambda)$ denotes the conductivity corresponding to the operator $\chi\left(H_{0}-\varrho\right) H_{0}$ and $\sigma_{p}(v, \lambda)$ for $\lambda \neq \varrho$ decreases, when $v \rightarrow 0$, faster than any power of $v$.

The main ideas how these facts can be proved are given at then end of this section, while the detailed arguments are given in Sects. 2 and 3. Our present 
intention is to discuss these facts in the framework of the spectral theory of random and almost periodic operators and from the viewpoint of the theory of disordered systems, the latter being a branch of theoretical physics were such mathematical studies find their applications.

Operator (1.1)-(1.7) is a continuous analog of the following finite difference operator acting in $\ell^{2}\left(\mathbb{Z}^{d}\right)$ :

$$
(h \psi)_{n}=\sum_{m \in \mathbb{Z}^{d}} w_{n-m} \psi_{m}+g \tan \pi[(\alpha, n)+\omega] \psi_{n},
$$

where $n \in \mathbb{Z}^{d}, w_{n}^{*}=w_{-n},\left|w_{n}\right| \leq C e^{-\theta|n|}, C, \theta>0$.

This operator was introduced in the physical paper [4] and then studied in the papers $[5,6]$. It was shown there that if the vector $\alpha$ satisfies Eq. (1.5), then the spectrum of the operator $h$ coincides with $\mathbb{R}$, is pure point and has multiplicity 1. The eigenvalues $\lambda_{m}, m \in \mathbb{Z}^{d}$, are in one-to-one correspondence with the $\mathbb{Z}^{d}$ points, and the respective eigenfunctions have the form

$$
\psi_{n}^{(m)}=\varphi_{n-m}(\lambda m)
$$

where $\varphi_{n}(\lambda), n \in \mathbb{Z}^{d}$, for every $\lambda \in \mathbb{R}$ decrease exponentially as $|n| \rightarrow \infty$.

In the one-dimensional case $(d=1)$ the absolutely continuous spectrum component is absent for all irrational $\alpha \in \mathbb{R}$.

The most natural continuous analog of the operator (1.10) seems to be given by the Schrödinger operator with a local potential

$$
q(x)=\sum_{n \in \mathbb{Z}^{d}} g \cdot t_{n} \cdot \delta_{d}(x-n) \quad x \in \mathbb{R}^{d},
$$

where $\delta_{d}(x), d=1,2,3$, is the so-called point potential (e.g., see [7]). For $d=1$ $\delta_{1}(x)$ is the one-dimensional Dirac delta-function; the spectral analysis of the Schrödinger operator in this case is reduced (by a simple trick) to the spectral analysis of the operator $h$ [8]. Thus, in the one-dimensional case the Schrödinger operator with the local potential (1.11) has a pure point spectrum, while the Schrödinger operator with the nonlocal potential (1.1) to (1.7), i.e. for $\varrho<\infty$ has, according to Theorem 1.1, an absolutely continuous component even for $d=1$. Besides, if the nonlocality is strong enough $\left(\varrho<\frac{1}{4}\right)$, then, according to Theorem 1.3 , an absolutely continuous component is present on the semiaxis $[\varrho, \infty)$ along with a dense point one, i.e. these two components coexist on $[\varrho, \infty)$.

It should be noted that in [19] a massive absolutely continuous component was found in a high energy part of the spectrum of the one-dimensional Schrödinger operator $H_{1}$ with a local smooth and quasiperiodic potential satisfying the Diophantine condition (1.5).

Moreover, results of recent papers [20-22] show that under certain additional conditions all spectrum on the semiaxis $\left[\lambda_{1}, \infty\right), \lambda_{1}$ being large enough, is absolutely continuous, but the low energy region [inf $\left.\operatorname{spec} H_{1}, \lambda_{2}\right), \lambda_{2} \ll \lambda_{1}$ is pure point. Thus, one should expect the transition from a pure point spectrum (localized states) to an absolutely continuous one (extended or delocalized states) in the intermediate region $\left[\lambda_{1}, \lambda_{2}\right]$, and the present state of our knowledge does not allow us to exclude mixing of these two components in the mentioned intermediate 
region $^{1}$. For a local potential the mechanism of the localization-delocalization transition is provided by quantum mechanical resonances [10, 20].

Our operator (1.1) to (1.7) shows that a nonlocality of a potential can supply some other mechanism of delocalization and coexistence of point and absolutely continuous spectra.

In the theoretical physics of disordered systems it is widely accepted that the vicinity of the spectrum lower boundary of the Schrödinger operator with random potential belongs to the point component (in the discrete case the vicinities of both boundaries belong to this component). This part of the spectrum of random operators was singled out and studied, on a physical level by I. M. Lifschitz $[7,8]^{2}$. He called this part of the spectrum the fluctuation one since its existence is due to large deviations (strong fluctuations) of the potential. These fluctuations are a system of deep potential wells located chaotically and far apart from one another.

The potential $g \cdot \tan \pi[(\alpha, n)+\omega], n \in \mathbb{Z}^{d}$, of the operator $h$ from Eq. (1.10) in the case of the Diophantine $\alpha$ 's has a similar structure. Thus, it is natural to expect that the spectrum of the operator $h$ studied in [4-6] as well as the behaviour of the respective low frequency conductivity (see [6]) model the fluctuation spectrum and conductivity behaviour for typical random potentials in their fluctuation spectrum (see also [11]).

An important question in the theory of disordered systems is stability of the point spectrum, in particular, the fluctuation spectrum (the Anderson localization) when the particle interaction is switched on. Convincing physical arguments are known suggesting that the interaction inhibits the localization (see, e.g., survey [12]). A well-known device in solid state physics to account for the interaction within the one particle approximation, is to introduce nonlinear or nonlocal terms in the Schrödinger equation (in the latter case the potential becomes a pseudopotential which, in the general case is an integral operator). The recent results by B. Soulliard and P. Devillard [13] brought evidence that nonlinearity inhibits the localization. The results of the present work on existence of the absolutely continuous spectrum for the simple essentially nonlocal ${ }^{3}$ pseudopotential (1.2)-(1.5), whose local variant (1.11) has a pure point spectrum, should, perhaps, be considered as another argument suggesting that the interaction inhibits the localization and enhances the appearance of extended states even in the fluctuation spectrum.

One should note, however, that the question seems to be not too simple, since in accordance with the recent papers $[14,15,18]$, switching on a small interaction retains at least a substantial portion of localized states.

\footnotetext{
1 It is widely believed that for the multidimensional $(d \geq 3)$ Schrödinger operator with a local random potential such mixing is impossible and the transition region is just a point, the socalled mobility edge [8]

2 The remarkable results recently obtained by J. Fröhlich and T. Spencer and their colleagues (see e.g. survey [10]) provided the status of mathematical theorems to many Lifschitz's insights; besides, they essentially extended and developed the fluctuation spectrum pattern outlined by I. M. Lifschitz 3 We mean that the Fourier transform of the function $v(x)$ in (1.2) has compact support [see (1.7)]. i.e., a parameter $\varrho$ which is a certain measure of nonlocality of the potential (1.2)-(1.5), is finite and even small
} 
Now we pass to the main points, actually to the algebraic essence of the proof of Theorems 1.1-1.3. The operator will be investigated by studying its resolvent $R(z)=(H-z)^{-1}$. It will be convenient to pass from the functions $\psi(x) \in L_{2}\left(\mathbb{R}^{d}\right)$ to their Fourier transform $\hat{\psi}(p)$, see (1.6). Introduce the operator $(T \psi)_{n}=t_{n} \psi_{n}$, $n \in \mathbb{Z}^{d}$, acting in $\ell^{2}\left(\mathbb{Z}^{d}\right)$. Here it is also convenient to pass to the discrete Fourier transform

$$
\hat{\psi}(p)=\sum_{n \in \mathbb{Z}^{d}} e^{2 \pi i(n, p)} \psi_{n}, \quad p \in \mathbb{T}^{d},
$$

where $\mathbb{T}^{d}=\mathbb{T}=\left[\frac{1}{2},-\frac{1}{2}\right]^{d}$. The cube $\mathbb{T}^{d}$ will often be replaced by the corresponding torus which will be denoted also by $\mathbb{T}^{d}$. Besides, we will often identify the functions $\psi$ on the torus $\mathbb{T}^{d}$ with the periodic functions $\psi(\cdot)=\psi(\cdot+n), n \in \mathbb{Z}^{d}$ in the entire space $\mathbb{R}^{d}$. Introduce a linear operator ()$_{\mathbb{T}}$ giving the correspondence between the function $\psi(p)$ and the periodic function $(\psi)_{\mathbb{T}}(p)$ :

$$
(\psi)_{\mathbb{T}}(p)=\sum_{n \in \mathbb{Z}^{d}} \psi(p+n), \quad p \in \mathbb{R}^{d} .
$$

Hence we obtain the following representation for $H$ :

$$
(H \varphi)(p)=p^{2} \varphi(p)+\hat{v}(p) T(\hat{v} \varphi)_{\mathbb{T}}(p) .
$$

After a simple algebra we get from (1.13)

$$
\begin{gathered}
(R(z) \varphi)(p)=\frac{\varphi(p)}{p^{2}-z}-\frac{\hat{v}(p)}{p^{2}-z}\left(T^{-1}+w_{z}\right)^{-1}\left(\frac{\hat{v} \varphi}{p^{2}-z}\right)_{\mathbb{T}}(p), \\
w_{z}(p)=\left(\frac{\hat{v}^{2}(p)}{p^{2}-z}\right)_{\mathbb{T}} .
\end{gathered}
$$

In (1.14) $w_{z}$ is a multiplication operator by the corresponding function. Note that equality (1.14) implies that the subspaces $\mathscr{H}_{ \pm}$reduce $H$ : the restriction of $H$ to $\mathscr{H}_{+}$coincides with the restriction of the operator $-(2 \pi)^{-2} \Delta$ onto this subspace, hence, relation (1.8) is valid.

To define the eigenfunctions $u_{\lambda}(p)$ and the respective eigenvalues $\lambda$ of the operator $H$, we will use (1.13), whence we get

$$
\left(p^{2}-\lambda\right) u_{\lambda}+\hat{v} T\left(\hat{v} u_{\lambda}\right)_{\mathbb{T}}=0 .
$$

Now it is clear that the eigenfunctions have the form

$$
u_{\lambda}(p)=\frac{\hat{v}(p)}{p^{2}-\lambda} g_{\lambda}(p), g_{\lambda}(p+n)=g_{\lambda}(p), \quad n \in \mathbb{Z}^{d},
$$

where the periodic function $g_{\lambda}$, as an element of $L_{2}\left(\mathbb{T}^{d}\right)$ satisfies the equation

$$
T^{-1} g_{\lambda}+w_{\lambda} g_{\lambda}=0 .
$$

The latter implies that $\lambda$ is an eigenvalue if and only if Eq. (1.17) has a nontrivial solution. For the sake of convenience we rewrite the operator $T^{-1}+w_{\lambda}$; to 
this end, we will introduce a unitary in $\ell_{2}\left(\mathbb{Z}^{d}\right)$ operator $U$ of multiplication by $\exp \{-2 \pi i(\alpha, n)\}$. Then

$$
\begin{gathered}
T^{-1}=i(I+\varkappa U)(I-\varkappa U)^{-1}, \quad \varkappa=e^{-2 i \pi \omega}, \\
(U \varphi)(p)=\varphi(p-\alpha) .
\end{gathered}
$$

Hence, after some algebra, we obtain (cf. [6])

$$
\begin{aligned}
T^{-1}+w_{\lambda} & =\left(w_{\lambda}+i I\right)\left(I-c_{\lambda} \varkappa V\right)(I-x V)^{-1}, \\
c_{\lambda}(p) & =\left(w_{\lambda}(p)-i\right)\left(w_{\lambda}(p)+i\right)^{-1} .
\end{aligned}
$$

Let us introduce the functions

$$
\begin{gathered}
f(\lambda, p)=\log c_{\lambda}(p), \\
f_{0}(\lambda)=\int_{\mathbb{T}^{d}} f(\lambda, p) d p=i 2 \pi m(\lambda), \quad m \in \mathbb{R}, \\
t(\lambda, p)=(I-U)^{-1}\left[f(\lambda, p)-f_{0}(\lambda)\right] .
\end{gathered}
$$

If the function $f(\lambda, p)$ is infinitely differentiable with respect to $p$ on the torus $\mathbb{T}$, then $t(\lambda, p)$ has the same property. It is easy to check by regarding $f$ and $t$ as elements of the space $\ell_{2}\left(\mathbb{Z}^{d}\right)$ and employing condition (1.3) which is important here. Equations (1.21)-(1.23) imply

$$
c_{\lambda} U=e^{f_{0}(\lambda)} e^{t(\lambda)} U e^{-t(\lambda)},
$$

where $c_{\lambda}$ and $t(\lambda)$ are operators of multiplication by the respective functions of the arguments $p$. Combining this result with (1.20), we obtain

$$
T^{-1}+w_{\lambda}=\left(w_{\lambda}+i I\right) e^{t(\lambda)}\left[I-e^{f_{0}(\lambda)} \varkappa U\right] e^{-t(\lambda)}(I-\varkappa U)^{-1} .
$$

Now, let

$$
e_{\lambda}=e^{-t(\lambda)}(I-\varkappa U)^{-1} g_{\lambda} .
$$

Then (1.25) implies that (1.17) is equivalent to the equation

$$
\left(I-e^{f_{0}(\lambda)} \varkappa U\right) e_{\lambda}=0 .
$$

From here it is not difficult to see that the eigenvalues $\lambda_{n}$ are the solutions of the equation

$$
m(\lambda)-\omega-(\alpha, n)=0(\bmod 1), \quad n \in \mathbb{Z}^{d},
$$

which is obtained with the aid of (1.22). For each $n$ Eq. (1.22) has no more than one solution $\lambda_{n}$ (so the spectrum multiplicity is 1 ) with the corresponding $e_{\lambda_{n}}=\exp \{2 \pi i(p, n)\}$. Combining this result with (1.26) and (1.23), we get

$$
\begin{aligned}
& g_{\lambda_{n}}(p)=(I-\varkappa U) e^{t\left(\lambda_{n}, p\right)} e^{2 \pi i(n, p)}, \\
& g_{\lambda_{n}}(p)=-2 i\left(w_{\lambda_{n}}(p)-i\right)^{-1} \exp \left\{t\left(\lambda_{n} p\right)+2 \pi i(p, n)\right\} .
\end{aligned}
$$

Substituting these values of $g_{\lambda_{n}}(p)$ to (1.16) we get the eigenfunctions.

To conclude the section we would point out that technically the central point is the proof of the smoothness of the function $f(\lambda, p)$. 


\section{Analytical Properties of Auxiliary Functions}

The present section is devoted to studying analytical properties of the functions $w_{z}(p), f(z, p), f_{0}(z)$ and $t(z, p)$. All properties of these functions important for further analysis are formulated and presented as lemmas. Everywhere below $z \in \mathbb{C}, z=\lambda+i \xi$.

Lemma 2.1. Let $\varrho>\frac{1}{4}$. Then

(a) $\operatorname{Im} z \operatorname{Im} w_{z}(p) \geq 0$; besides for $p^{2}<\varrho$ if $\operatorname{Im} z \neq 0$, then $\operatorname{Im} w_{z}(p) \neq 0$.

(b) There exists a sufficiently small positive $\delta$, such that for every $z$ from the strip $L_{\delta}=\{z \in \mathbb{C}, \operatorname{Re} z<\delta,-|\operatorname{Im} z|<\delta\}$ the function $w_{z}(p), p \in \mathbb{T}^{d}$, assumes values from the following subset of $\mathbb{C}$ :

$$
\{|\zeta| \geq 2\} \cup K, K=\{|\operatorname{Im} \zeta| \leq \operatorname{Re} \zeta\} .
$$
tion

(c) One may choose a continuous branch $\log (\cdot)$ so that $\log 1=0$ and the func-

$$
f(z, p)=\log c_{z}(p), \quad c_{z}(p)=\frac{w_{z}(p)-i}{w_{z}(p)+i}
$$

is well-defined for $z \in L_{\delta}, p \in \mathbb{T}^{d}$, and

$$
f(\lambda, p)=-2 i \operatorname{Arccot} w_{\lambda}(p),
$$

where for $\operatorname{Arc} \cot (\cdot)$ a branch is chosen which is continuous at infinity and is determined by the relations

$$
\operatorname{Arccot}( \pm \infty)=0, \operatorname{Arccot}( \pm 0)= \pm \frac{\pi}{2} .
$$

(d) $f(z, p)$ is an infinitely differentiable function in $\lambda, \xi$ and $p$ in the domain $\mathscr{L}_{\delta}=L_{\delta} \times \mathbb{T}^{d}$ and is analytical with respect to $z \in L_{\delta}$ :

(e) The function

$$
f_{0}(z)=\int_{\mathbb{T}} f(z, p) d p
$$

is analytical with respect to $z \in L_{\delta}$ and satisfies the relations

$$
\begin{aligned}
\pm \operatorname{Re} f_{0}(z)>0, & \text { if } \quad \pm \operatorname{Im} z<0, \\
\operatorname{Re} f_{0}(z)=0, & \text { if } \quad \operatorname{Im} z=0, \\
f_{0}(\lambda)=2 \pi i m(\lambda), m(\lambda)= & -\frac{1}{\pi} \int_{\mathbb{T}^{d}} \operatorname{Arccot} w_{\lambda}(p) d p,
\end{aligned}
$$

$m(\lambda), \lambda<\delta$, being a monotonically increasing function of $\lambda$ with values lying in the interval $\left(-\frac{1}{2}, \frac{1}{2}\right)$ and $m(-\infty)=-\frac{1}{2}$.

(f) The function $m^{\prime}(\lambda)=\frac{\partial}{\partial \lambda} m(\lambda)$ can be represented as

$$
\begin{gathered}
m^{\prime}(\lambda)=\frac{1}{\pi} \int_{\mathbb{R}^{d}}|\ell(\lambda, p)|^{2} d p, \quad \lambda<\delta, \\
\ell(\lambda, p)=\frac{\hat{v}(p)}{p^{2}-\lambda}\left(w_{\lambda}(p)+i\right)^{-1} .
\end{gathered}
$$


(g) There exists a function $t(z, p)$ satisfying the homological equation

$$
t(z, p)-t(z, p-\alpha)=f(z, p)-f_{0}(z), \quad p \in \mathbb{T}^{d},
$$

which is infinitely differentiable in its arguments $\lambda, \xi$ and $p$ in the domain $\mathscr{L}_{\delta}$ and is analytical with respect to $z \in L_{\delta}$. Besides, for real $\lambda$,

$$
\operatorname{Re} t(\lambda, p) \equiv 0, \quad p \in \mathbb{T}^{d} .
$$

Proof. We will need the following representations of the function $w$ which directly follow from definition (1.15):

$$
\begin{aligned}
& w_{z}(p)=\sum_{n \in \mathbb{Z}^{d}} \frac{\hat{v}^{2}(p+n)\left((p+n)^{2}-\lambda\right)}{\left((p+n)^{2}-\lambda\right)^{2}+\xi^{2}}+i \sum_{n \in \mathbb{Z}^{d}} \frac{\hat{v}^{2}(p+n) \xi}{\left((p+n)^{2}-\lambda\right)^{2}+\xi^{2}}, \\
& w_{z}(p)=\frac{\hat{v}^{2}(p)}{p^{2}-z}+w_{z}^{(1)}(p), w_{z}^{(1)}(p)=\sum_{n \neq 0} \frac{\hat{v}(p+n)}{\left(p+n\left(^{2}-z\right.\right.} .
\end{aligned}
$$

Since the function $w$ is periodic in $p$, we will assume in what follows that

$$
\left|p_{j}\right| \leq \frac{1}{2}, \quad 1 \leq j \leq d .
$$

The validity of assertion (a) immediately follows from (2.11).

To demonstrate (b) we will remark, at first, that

$$
(p+n)^{2} \geq \max _{1 \leq j \leq d}\left|p_{j}+n_{j}\right|^{2} \geq \frac{1}{4}, \quad n \neq 0 .
$$

From here and from the definition of the function $w^{(1)}$ it follows that at $\lambda<\frac{1}{8}$,

$$
\left|w^{(1)}(p)\right| \leq \sum_{n \neq 0} \frac{\hat{v}^{2}(p+n)}{\frac{1}{4}-\lambda} \leq A_{1}
$$

where $A_{1}$ is a constant independent of $\lambda$; the boundedness of $A_{1}$ is ensured by Condition $V$. Introduce a pair of arbitrary (for the time being) constant $\delta$ and $\delta_{1}, 0<\delta<\delta_{1}$, and consider the following sets of pairs $\langle z, p\rangle$ :

$$
\begin{aligned}
\mathfrak{U}_{+} & =\mathscr{L}_{\delta} \cap\left\{\langle z, p\rangle: p^{2}-\lambda>\delta\right\}, \\
\mathfrak{U} & =\mathscr{L}_{\delta} \cap\left\{\langle z, p\rangle:\left|p^{2}-\lambda\right|<\delta_{1}\right\} .
\end{aligned}
$$

Show that

$$
\mathscr{L}_{\delta}=\mathfrak{U} \cup \mathfrak{U}_{+} \cdot
$$

Indeed, let $\langle z, p\rangle \in \mathscr{L}_{\delta}$ and $\langle z, p\rangle \bar{\in} \mathfrak{A}$. It is easy to see that $p^{2}-\lambda$ is positive, since otherwise $p^{2}-\lambda \leq-\delta_{1}$, i.e. $\lambda>\delta_{1}>\delta$. Hence, $p^{2}-\lambda \geq \delta_{1}>\delta$, i.e. $\langle z, p\rangle \in \mathfrak{U}_{+}$ which terminates the demonstration of the validity of (2.12).

Let us check now, for $\delta<\frac{1}{8}$ the validity of the relation

$$
\langle z, p\rangle \in \mathfrak{U}_{+} \Rightarrow w_{z}(p) \in K,
$$

where the cone $K$ is defined in point (b) of the Lemma. To this end, we make use of (2.11) which implies

$$
\delta^{-1} \operatorname{Re} w_{z}(p) \geq \sum_{n} \frac{\hat{v}^{2}(p+n)}{\left((p+n)^{2}-\lambda\right)^{2}+\xi^{2}} \geq \delta^{-1}\left|\operatorname{Im} w_{z}(p)\right|,
$$


where the left inequality is obtained from inequality (2.14). It immediately follows from inequalities (2.18) that $w_{z}(p) \in K$.

Let us examine the case $\langle z, p\rangle \in \mathfrak{U}$ now and show that for a sufficiently small $\delta$

$$
\left|w_{z}(p)\right| \geq 2,\langle z, p\rangle \in \mathfrak{A} \text {. }
$$

Introduce the constant $a_{1}=\min _{p^{2} \leq \frac{1}{4}} \hat{v}^{2}(p)$ which is positive from the condition of the lemma $\varrho>\frac{1}{4}$ and Condition $V$. Combining relations (2.12) and (2.15), we obtain for $\langle z, p\rangle \in \mathfrak{U}$ and $\delta<\frac{1}{8}$,

$$
\left|w_{z}(p)\right|>\left|\delta_{1}+i \xi\right|^{-1} \min _{p^{2} \leq \delta+\delta_{1}} \hat{v}^{2}(p)-A_{1} \geq \frac{a_{1}}{2 \delta_{1}}-A_{1} .
$$

Hence, assuming that the following inequalities

$$
0<\delta<\delta_{1} \min \left\{\frac{1}{8}, a_{1} / 2\left(2+A_{1}\right)\right\}
$$

are valid, we obtain (2.19). Thus, for $\delta$ satisfying (2.20) both (2.17) and (2.19) are valid; the latter relations together with (2.16) warrant the validity of point (b) of the lemma.

In order to demonstrate (c) and (d) we make use of the obvious inequalities for complex $w$ :

$$
\begin{array}{lll}
\operatorname{Re}(w-i)(w+i)^{-1}>0, & \text { if } & |w|>1, \\
\operatorname{Im}(w-i)(w+i)^{-1}<0, & \text { if } & \operatorname{Re} w>0 .
\end{array}
$$

These inequalities by virtue of point (b) of the lemma imply the following relations for the function $c_{z}$ defined in (2.1):

$$
c_{z}(p) \in\{\operatorname{Re} \zeta>0\} \cup\{\operatorname{Im} \zeta<0\}, \quad A_{2}^{-1} \leq\left|c_{z}(p)\right| \leq A_{2},
$$

with $A_{2}$ being a positive constant. Hence, the function $f(z, p)$ can be obviously defined by relations (2.1) from which equality (2.2) immediately follows. That the function $f$ is smooth and analytical in the corresponding variables in the domain $\mathfrak{A}_{+}$follows from similar properties which follow from representation (2.11). The same properties of the function $f$ in the domain $\mathfrak{A}$ can be easily demonstrated through multiplying both the numerator and the denominator of the fraction defining $c_{z}$ by $p^{2}-z$ and making use of relations (2.12), (2.15), and (2.20). It remains to note that both $\mathfrak{A}$ and $\mathfrak{U}_{+}$are open sets whose union coincides with $\mathscr{L}_{\delta} ;$ this terminates the proof of points (c) and (d).

Examining the points (e) and (f), note that the analyticity of the function $f_{0}(z)$ directly follows from its definition by equality (2.4) and from point (d). Further we will use the following elementary relations implied by $(2.1)$ :

$$
\operatorname{Re} f \cdot \operatorname{Im} w \leq 0 ; \quad \operatorname{Re} f=0, \quad \text { if } \quad \operatorname{Im} w=0 .
$$

Combining this result, definition (2.4) and point (a) of the lemma, we prove the validity of relations $(2.5)$ to $(2.8)$, as well as other assertion of point (e). As to 
point $(\mathrm{g})$, note, first of all, that the function $t(z, p)$ whose Fourier coefficients $t_{n}(z), n \in \mathbb{Z}^{d}$, are given by the equalities

$$
\begin{aligned}
& t_{n}(z)=\left(1-e^{-2 \pi i(\alpha, n)}\right)^{-1} f_{n}(z), \quad n \neq 0, \\
& t_{0}(z)=0,
\end{aligned}
$$

where $f_{n}$ are the Fourier coefficients of the function $f$, at least formally satisfies Eq. (2.9). But, by virtue of point (d) the Fourier coefficients $f_{n}(z)$ are analytical with respect to $z$ and uniformly in $z \in L_{\delta}$ decrease faster than any power of $|n|^{-1}$. Combining this result, the Diophantine condition (1.3) and (2.23), one easily concluded that the coefficients $t_{n}(z)$ possess the same properties. Therefore, $t(z, p)$ is smooth and analytical in the corresponding variables and it satisfies Eq. (2.9). Relation (2.10) immediately follows from (2.22) and (2.23). The lemma is proved.

Lemma 2.2. Let $\varrho<\frac{1}{4}$. Then (a) the function $w_{z}(p)$ is equal to $\hat{v}^{2}(p)\left(p^{2}-z\right)^{-1}$ inside the ball $B=\left\{p \in \mathbb{T}^{d}: p^{2} \leq \varrho\right\}$ and the zero outside the ball, i.e. on the set $B^{c}=\mathbb{T}^{d} \backslash B ; \operatorname{Im} z \cdot \operatorname{Im} w_{z}(p) \geq 0$.

(b) Extend the function a $(\mu)$ from Condition $V$ for negative $\mu$ by the constant $a(0)$ and introduce the complex sets

$$
\begin{aligned}
L & =L_{1} \cap\{\operatorname{Re} \zeta \geq \varrho\} \supset \mathbb{R}, \\
L_{1} & =\left\{\zeta \in \mathbb{C}: \operatorname{Re} \zeta<\varrho,|\operatorname{Im} \zeta|<\frac{1}{2} a(\operatorname{Re} \zeta),\right. \\
\frac{1}{2} & \left.\leq\left|\frac{\zeta-\mu-i a(\mu)}{\zeta-\mu+i a(\mu)}\right| \leq 2 \text { for } 0 \leq \mu \leq \varrho\right\},
\end{aligned}
$$

then the function $c_{z}(p)$ defined in (2.1), assumes no negative real values for $\langle z, p\rangle \in$ $L \times B$, and for some positive constant $C$,

$$
C^{-1} \leq\left|c_{z}(p)\right| \leq C,\langle z, p\rangle \in L \times \mathbb{T}^{d}
$$

$L_{1}$ is an open set containing the ray $(-\infty, \varrho)$.

(c) Let $\log (\cdot)$ be a continuous branch given on the complex plane with a cut on the negative real half-axis, $\log 1=0$, then relations (2.1) define the function $f$ on the set $L \times B$; outside of this set assume by definition that

$$
f(z, p)=i\left\{\begin{array}{rl}
-\pi, & \operatorname{Re} z<\varrho \\
\pi, & \operatorname{Re} z \geq \varrho
\end{array}, \quad p^{2}>\varrho\right.
$$

with relations (2.2) and (2.3) being valid for any real $\lambda$ and $\varrho$ on the torus $\mathbb{T}^{d}$;

(d) $f(z, p)$ is an infinitely differrentiable function in the arguments $\lambda$, $\xi$ and $p$ in the internal points of the set $L \times \mathbb{T}^{d} \backslash \mathscr{P}$, where $\mathscr{P}=\left\{\langle z, p\rangle: \lambda=\varrho, p^{2} \geq \varrho\right\}$; $f$ is an analytical function in $z$ in the internal points of $L$ and has a discontinuity defined by equality (2.25) on the surface $\mathscr{P} ; f(z, p)$ is infinitely differentiable up to the boundary in the arguments $\lambda, \xi$ and $p$ in the domain $\{\operatorname{Re} z \geq \varrho\} \times \mathbb{T}^{d}$.

(e) The function $f_{0}(z)$ defined on $L$ by equality (2.4) is analytical in the internal points of the set $L$ and is infinitely differentiable up to the boundary on the set $\{\operatorname{Re} \zeta \geq \varrho\}$, satisfies the relations (2.5) and (2.6), where $m(\lambda), \lambda \in \mathbb{R}$, is a monotonically increasing, continuous from the right, real analytical at $\lambda \neq \varrho$ function; 
here $m( \pm \infty)= \pm \frac{1}{2}, m(\varrho)-m(\varrho-0)=\left(1-V_{\varrho}\right)$, where $V_{\varrho}$ is the volume of the $d$-dimensional ball of radius $\varrho$.

(f) The function $m^{\prime}(\lambda)=\partial m / \partial \lambda$ can be represented as

$$
\begin{aligned}
m^{\prime}(\lambda) & =\pi^{-1} \int_{p^{2} \leq \varrho}|\ell(\lambda, p)|^{2} d p, \\
\ell(\lambda, p) & =\hat{v}(p)\left(p^{2}-\lambda+i \hat{v}^{2}(p)\right),
\end{aligned}
$$

where $m^{\prime}(\lambda)$ is regarded as the right derivative and

$$
\int_{-\infty}^{\infty} m^{\prime}(\lambda) d \lambda=V_{\varrho}
$$

(g) There exists a function $t(z, p)$ defined on the set $L \times \mathbb{T}^{d}$ that satisfies the homological Eq. (2.9), is infinitely differentiable in the arguments $\lambda, \xi$ and $p$ in the internal points of the set $L \times \mathbb{T}^{d} \backslash \mathscr{P}$, is infinitely differentiable up to the boundary on the set $\{\operatorname{Re} \zeta \geq \varrho\} \times \mathbb{T}^{d}$, is analytical in $z$ in the internal points of the set $L$, is infinitely differentiable in $p$ for any $z$ from $L$, i.e. including all real $z$; for the function $t(\lambda, p), \lambda \in \mathbb{R}$, relation (2.10) holds.

Proof. Note first of all that the assertions of this lemma are similar to the appropriate assertion of Lemma 2.1. The only difference is that here the functions $c_{z}(p), f(z, p)$ and $f_{0}(z)$ are defined on a broader, as to $z$, set $L \supset \mathbb{R}$ so, thanks to the condition $\varrho<\frac{1}{4}$, one can study the properties of these functions on a broader set $L \times \mathbb{T}^{d}$. With this similarity in mind, we will skip reasoning common for both lemmas.

Since $\varrho<\frac{1}{4}$, the operator $(\cdot)_{\mathbb{T}}$ in the definition on $w$ in $(1.15)$ may be omitted, which immediately demonstrates point (a). Combining this with Condition $V$, we obtain the following representation:

$$
\begin{aligned}
& c_{z}(p)=-\frac{b\left(p^{2}\right)-z}{b^{*}\left(p^{2}\right)-z}, b(\mu)=\mu+i a^{2}(\mu), \quad p \in B, \\
& c_{z}(p)=-1, \quad p \in B^{c}, \quad z \in L .
\end{aligned}
$$

Introducing the set $L$ in point (b) we, having in mind to define the function $f=\log c$, must ensure the following: 1) $L \supset \mathbb{R}$; 2) for $z \in L: z \neq b(\mu), b^{*}(\mu)$, $\mu \in \mathbb{R}$, and in $L$ there exists no closed contour surrounding the points $b(\mu)$ and $b^{*}(\mu)$; 3) for $p \in B$ the function $c_{z}(p)$ does not assume values on the real negative half-axis; 4) smoothness of the function $c$ on a sufficiently broad set. It is easy to check whether the first three requirements are met on the set $L$. Smoothness and analyticity of the function $c$ in the appropriate arguments on the set $\left\{|\operatorname{Im} \zeta| \leq \frac{1}{2} a(\operatorname{Re} \zeta), \operatorname{Re} \zeta<\varrho\right\} \times \mathbb{T}^{d}$ immediately follow from (2.9). The infinite differentiability of the function $c$, hence $f$, up to the boundary on the set $\{\operatorname{Re} \zeta \geq \varrho\} \times \mathbb{T}^{d}$ follows from the inequality

$$
|c+1|=\frac{2|\operatorname{Im} b|}{\left|b^{*}-z\right|} \leq \frac{2 a^{2}\left(p^{2}\right)}{\left|p^{2}-\varrho\right|}, \quad p^{2}<\varrho, \operatorname{Re} z \geq \varrho,
$$


since Condition $V$ implies that, when $\mu \rightarrow \varrho-0 a(\mu)$ tends to zero faster than any power of $\varrho-\mu$. Inequality (2.24) follows from the definition of the set $L_{1}$ and inequality (2.30). Relation (2.25) can be easily deduced from (2.29).

As to the properties of the function $f_{0}(z)$, it is smooth and analytical as well as $f(z p)$, the properties of the latter being valid for the former since it is defined by Eq. (2.4); the jump of the function $m(\lambda)$ at the point $\varrho$ is calculated from relations (2.25), whence we get Eq. (2.28).

Finally, the assertion of point (g) is proved similarly to the corresponding point of Lemma 2.1, using point (d) of the present lemma.

\section{Selfadjointness and Structure of the Spectrum}

The present section contains proofs of Theorems 1.1-1.3 together with important representations for the resolvent and eigenfunctions of the operator $H$.

Lemma 3.1. Let $H$ be the operator by Eqs. (1.1) to (1.7): Then $H S \subset S$ and $H$ is a symmetric operator on $S$.

Proof. The Diophantine condition (1.5) implies the inequality

$$
\left|t_{n}\right| \leq C_{1}\left(|n|^{\beta}+1\right)^{-1}, \quad n \in \mathbb{Z}^{d},
$$

where $C_{1}$ is a positive constant while it follows from Condition $V$ that $v \in S$ and $\forall \psi \in S, B>0 \exists C(\psi, B)$ :

$$
\left|\left(v_{n}, \psi\right)\right| \leq C(\psi, B)(|n|+1)^{-B}, \quad n \in \mathbb{Z}^{d} .
$$

It is not difficult to observe that the two latter inequalities together with the relations $v_{n} \in S$ imply that

$$
Q \psi=\sum_{n \in \mathbb{Z}^{d}} t_{n}\left(v_{n}, \psi\right) v_{n} \in S,
$$

the series being convergent in the metrics of the space $S$. Hence, taking into account the obvious relation $\Delta S \subset S$, we conclude that $H$ is a symmetric operator on $S$ that transforms the space $S$ into itself. The lemma is proved.

Lemma 3.2. Let for a fixed $z w_{z}(p), c_{z}(p)$ and $t(z, p)$ be functions on the torus $\mathbb{T}^{d}$ which are defined by relations (1.15), (2.1), and (2.9), while $w_{z}, c_{z}$ and $t(z)$ are operators of multiplication by the corresponding functions in the space $L_{2}\left(\mathbb{T}^{d}\right)$. We shall assume that in the notation of Lemmas 2.1 and $2.2 z \in L_{\delta}$, if $\varrho>\frac{1}{4}$, and $z \in L$, if $\varrho<\frac{1}{4}$. Then the following representations and an inequality are valid:

$$
\begin{gathered}
c_{z} V=e^{f_{0}(z)} E^{t(z)} U e^{-t(z)}, \\
T^{-1}+w_{z}=\left(w_{z}+i I\right) e^{t(z)}\left(I-e^{f_{0}(z)} \varkappa V\right) e^{-t(z)}(I-\chi V)^{-1}, \quad \operatorname{Im} z \neq 0, \\
\left\|\left(T^{-1}+w_{z}\right)^{-1}\right\| \leq\left|C_{2}\right| 1-\left.e^{\operatorname{Re} f_{0}(z)}\right|^{-1}, \quad \operatorname{Im} z \neq 0,
\end{gathered}
$$

where, in the case $\varrho>\frac{1}{4}, C_{2}$ is a positive constant independent of $z \in L_{\delta}$, while in the case $\varrho<\frac{1}{4}$, if $A$ is such a bounded closed interval that $A \subset(-\infty, \varrho)$ or $A \subset[\varrho,+\infty)$, then $C_{2}=C_{2}(A)$ for $z \in L \cap\{\operatorname{Re} \zeta \in A\}$. 
Proof. Equation (3.4) immediately follows from definition (1.19) of the operator $U$ and Lemmas 2.1, 2.2(g): To establish Eq. (3.5) one has to use (1.18) for obtaining (1.20), exchanging $\lambda$ for $z$, and then to substitute the right-hand-side of Eq. (3.4) instead of $c_{z} U$. Since for $\operatorname{Im} z \neq 0 w_{z}$ is a bounded operator, the domains of both sides of (3.5) and of the operator $(I-\chi U)^{-1}$ all coincide because of (1.18).

Finally, in order to obtain inequality (3.6) we make use of representation (3.5), whence we obtain

$$
\left(T^{-1}+w_{z}\right)^{-1}=(I-\varkappa U) e^{t(z)}\left(I-e^{f_{0}(z)} \varkappa U\right)^{-1} e^{t(z)}\left(w_{z}+i I\right)^{-1} .
$$

It is obvious that

$$
\|I-\chi U\|=2,\left\|\left(I-e^{f_{0}(z)} \chi U\right)^{-1}\right\|=\left|1-e^{\operatorname{Re} f_{0}(z)}\right|^{-1} .
$$

Besides, the inequalities hold,

$$
\left\|\left(w_{z}+i\right)^{-1}\right\|=\frac{1}{2}\left\|c_{z}-1\right\| \leq C_{3}, \quad\|t(z)\| \leq C_{4} ;
$$

the former follows from (2.21) and (2.24), the latter follows from Lemmas 2.1 and 2.2(g). Equations (3.7)-(3.9) imply (3.6) which terminates the proof of the lemma.

Lemma 3.3 Let $z$ assume the same values that in the previous lemma with $\operatorname{Im} z \neq 0$, let $R(z)$ be an operator defined by Eq. (1.14). Then

(a) $R(z)$ is a bounded operator and $R(z) S \subseteq S$;

(b) $(H-z I) R(z) \varphi=\varphi, \forall \varphi \in S$;

(c) $H$ is an essentially self-adjoint operator on $S$ whose resolvent coincides with the operator $R(z)$;

(d) the spaces $\mathscr{H}_{ \pm}$reduce the operator $H$ namely

$$
\left.H\right|_{\mathscr{H}_{+}}=-\left.(2 \pi)^{-2} \Delta\right|_{\mathscr{H}_{+}} .
$$

Proof. Note first of all the following simple relations:

$$
\begin{aligned}
\psi_{1}(p+n) & =\psi_{1}(p), n \in \mathbb{Z}^{d} \Rightarrow\left(\psi \psi_{1}\right)_{\mathbb{T}}=(\psi)_{\mathbb{T}} \psi_{1}, \\
\int_{\mathbb{T}}\left|(g \psi)_{\mathbb{T}}(p)\right|^{2} d p & \leq \int_{\mathbb{T}}\left(\sum_{n}|g(p+n)|^{2}\right)\left(\sum_{n}|\psi(p+n)|^{2}\right) d p \\
& \leq \sup _{p \in \mathbb{T}}\left(|g|^{2}\right)_{\mathbb{T}}(p) \int_{\mathbb{R}^{d}}|\psi(p)|^{2} d p .
\end{aligned}
$$

Then the boundedness of the operator $R(z)$ immediately follows from Eqs. (3.11), (3.6), and (2.5). Choose an arbitrary $\varphi \in S$ and consider $R(z) \varphi$. The first term in (1.14) obviously belongs to $S$. To establish the same for the second term, make use of (3.7) and note the validity of the following relation:

$$
\forall \varphi \in S:\left(T^{-1}+w_{z}\right)^{-1}\left(\frac{\hat{v} \varphi}{p^{2}-z}\right)_{\mathbb{T}} \in C^{\infty}(\mathbb{T}),
$$

which follows from the identity $\left(w_{z}+i\right)^{-1}=\frac{1}{2} i c_{z}=\frac{i}{2} \exp \{f(z)\}$ Lemmas 2.1, 2.2 (d.g) and Eq. (2.5). It is obvious that $v \in S$, so, because of (3.12) the second term in (1.14) belongs to $S$, hence, $R(z) S \subseteq S$. Point (a) is proved. 
The assertion of point (b) of the lemma is checked directly, by using point (a) and Eq. (3.10). Points (b) and (a) immediately imply that $(H-z I) S=S$, whence it follows that the operator $H$ is essentially self-adjoint on $S$, [16]. Combining this assertion and (b), we easily obtain the validity of the second assertion of point (c).

Finally, to prove (d) from (a) to (c), it suffices to make sure that the spaces $\mathscr{H}_{ \pm}$coinciding with the functions from $L_{2}\left(\mathbb{R}^{d}\right)$ with supports in the sets $\left\{ \pm p^{2}> \pm \varrho\right\}$ reduce the resolvent $R(z)$ and its action on $\mathscr{H}_{+}$coincides with that of the operator $\left(-(2 \pi)^{-2} \Delta-z\right)^{-1}$. The latter assertion can be easily deduced from representation (1.14) and Conditions $V$ which completes the proof of the lemma.

This lemma obviously implies Theorem 1.1.

Lemma 3.4. Let $\varrho<\frac{1}{4}$ and $\mathscr{M}=\left(-\frac{1}{2}, m(\varphi-0)\right) \cup\left[m(\varrho)\right.$, $\left.\frac{1}{2}\right]$, where the function $m(\lambda)$ was defined in Lemma 2.2(e). Then

(a) the set of eigenvalues of the operator $H$ coincides with the set of solutions of Eq. (1.28), the latter having exactly one solution for each $n$ if

$$
(\alpha, n)+\omega \in \mathscr{M}(\bmod 1),
$$

and no solutions if (3.13) does not hold;

(b) for each $n$ satisfying relation (3.13) there exists the eigenvalue $\lambda$ and the eigenfunction $u_{\lambda}$ of the operator $H$, such that

$$
\begin{aligned}
m\left(\lambda_{n}\right) & =\omega+(\alpha, n)(\bmod 1) \\
u_{\lambda_{n}}(p) & =\frac{\hat{v}(p)}{p^{2}-\lambda_{n}}(-2 i)\left(\frac{\hat{v}^{2}(p)}{p^{2}-\lambda_{n}}-i\right)^{-1} e^{t\left(\lambda_{n}, p\right)} e^{2 \pi i(n, p)}
\end{aligned}
$$

with $u_{\lambda_{n}} \in S$.

Proof. Assume that $\lambda$ and $u_{\lambda} \in L_{2}\left(\mathbb{R}^{d}\right)$ are eigenvalues and eigenvectors of the operators $H$, i.e. $H u_{\lambda}=\lambda u_{\lambda}$. The latter equality, because of the preceding lemma is equivalent to the equality $R(z) u_{\lambda}=(\lambda-z)^{-1} u_{\lambda}$ for every $z \in L \cap\{\operatorname{Im} \zeta \neq 0\}$. This equality, using (1.14) and some elementary algebra, can be rewritten in the form

$$
\begin{aligned}
& u_{\lambda}(p)=\hat{v}(p)\left(p^{2}-\lambda\right)^{-1} g_{\lambda}(p), \\
& g_{\lambda}(p)=-(\lambda-z)\left(T^{-1}+w_{z}\right)^{-1}\left(\hat{v}(p)\left(p^{2}-z\right)^{-1} u_{\lambda}(p)\right)_{\mathbb{T}} .
\end{aligned}
$$

Let us show that

$$
w_{\lambda} g_{\lambda}, \quad g_{\lambda}, \quad T^{-1} g_{\lambda} \in L_{2}(\mathbb{T}) .
$$

Indeed, by multiplying both sides of Eq. (3.16) by $\hat{v}(p)$ and applying the operation $(\cdot)_{\mathbb{T}}$ to the result, we obtain, on taking account of (1.15) and (3.10), that $w_{\lambda} g_{\lambda}=\left(v u_{\lambda}\right)_{\mathbb{T}}$. Hence, on using inequality (3.11), we obtain that $\left\|w_{\lambda} g_{\lambda}\right\|_{L_{2}(\mathbb{T})} \leq$ $\max _{p}|\hat{v}(p)|\left\|u_{\lambda}\right\|_{L_{2}\left(\mathbb{R}^{d}\right)}$. But the norm of the vector $u_{\lambda}$ has been assumed to be finite, hence, $w_{\lambda} g_{\lambda} \in L_{2}(\mathbb{T})$. Further, let us consider Eq. (3.17), noting that the operator $\left(T^{-1}+w_{z}\right)^{-1}$ because of inequality (3.6), is bounded, so the vector acted upon, by virtue of (3.11) belongs to $L_{2}(\mathbb{T})$. Therefore, $g_{\lambda} \in L_{2}(\mathbb{T})$. That is why Eq. (3.17) 
implies that $\left(T^{-1}+w_{\lambda}\right) g_{\lambda} \in L_{2}(\mathbb{T})$, whence, since the operator $w_{\lambda}$ is bounded, $T^{-1} g_{\lambda} \in L_{2}(\mathbb{T})$.

If one substitutes now expression (3.16) into Eq. (3.17) and, using relations (3.18), carries out some elementary transformations, one gets Eq. (1.17). Let us introduce the vector $\tilde{e}_{\lambda}=(I-\varkappa U)^{-1} g_{\lambda}$, which by virtue of (3.18) belongs to $L_{2}(\mathbb{T})$. Having applied the bounded operator $\left(w_{\lambda}+i I\right)^{-1}$ to both sides of Eq. (1.17) and using the obvious boundedness of the operator $w_{\lambda}\left(w_{\lambda}+i I\right)^{-1}$ we easily get

$$
\left[I-\left(w_{\lambda}-i I\right)\left(w_{\lambda}+i I\right)^{-1} \varkappa U\right] \tilde{e}_{\lambda}=0 .
$$

Combining this equality with Eqs. (3.4) and (2.9) at $z=\lambda$ we obtain equality (1.27), whence, by the same reasoning as in Sect. 1 , we get $\lambda_{n}$ and $u_{\lambda_{n}}$. The latter belong to the set $S \cap \mathscr{H}_{-}$, which follows from representation (3.15) and Lemma 2.2. It is not difficult to check with the aid of Eqs. (3.16), (3.17), (3.19), and (1.17), that they are eigenvalues and eigenfunctions. The lemma is proved.

Lemma 3.5. Let $\varrho<\frac{1}{4}$. Almost everywhere in $\omega \in[0,1)$ with respect to the Lebesgue measure the eigenfunctions of the preceding lemma form a basis of the space $\mathscr{H}_{-}$.

Proof. The functions $u_{\lambda_{n}}$ being the eigenfunctions of the selfadjoint operator $H$, are mutually orthogonal for various $n$. By normalizing them, we will introduce the functions

$$
\begin{aligned}
\tilde{u}_{n}(p) & =u_{\lambda_{n}}(p)\left\|u_{\lambda_{n}}\right\|^{-1} \\
& =\left(m^{\prime}\left(\lambda_{n}\right)\right)^{-\frac{1}{2}} \ell\left(\lambda_{n}, p\right) e^{t\left(\lambda_{n}, p\right)} e^{2 \pi i(n, p)} .
\end{aligned}
$$

Here we have used representation (1.30) and relations (2.10), (2.26), and (2.27). Let us introduce the projection-valued function

$$
\mathscr{I}_{p}(\lambda)=\sum_{\lambda_{n}<\lambda}\left|\tilde{u}_{n}\right\rangle\left\langle\tilde{u}_{n}\right|,
$$

where $\left|\tilde{u}_{n}\right\rangle\left\langle\tilde{u}_{n}\right|$ is an orthogonal projection onto the appropriate vector. $\mathscr{I}_{p}(\lambda)$ is, obviously, a nondecreasing function of $\lambda$ generating a projection-valued measure that will be denoted as $\mathscr{I}_{p}(d \lambda)$. We will choose an arbitrary $\varphi \in \mathscr{H}_{-}$and, having introduced the notation $\int_{0}^{1} \ldots d \omega=\langle\ldots\rangle$, we will investigate the nondecreasing function

$$
\left\langle\left(\varphi, \mathscr{I}_{p}(\lambda) \varphi\right)\right\rangle=\sum_{n}\left\langle\chi_{\lambda}\left(\lambda_{n}\right)\left|\left(\varphi, \tilde{u}_{n}\right)\right|^{2}\right\rangle
$$

with $\chi_{\lambda}$ being the characteristic function of the interval $(-\infty, \lambda)$. Consider a term with the subscript $n$ in the last sum and note that, because of representation (3.20), the dependence $\tilde{u}_{n}$ on the parameter $\omega$ is realized via the dependence of the eigenvalue $\lambda_{n}(\omega)$ on $\omega$. Here Lemma 3.4 implies that if $(\alpha, n)+\omega \bar{\epsilon} \mathscr{M}$ (mod) then the function $\tilde{u}_{n}(\omega, p)$ "disappears" from the set of the eigenfunctions of the operator $H$. It means, bearing in mind the right-hand side of Eq. (3.22), that for 
such $\omega$ 's $\left(\varphi, \tilde{u}_{n}\right)$ should be set to 0 . This remark, combined with Eq. (3.14) and equality (3.20), yield

$$
\begin{aligned}
\left.\left.\left\langle\chi_{\lambda}\left(\lambda_{n}\right)\right| \varphi, \tilde{u}_{n}\right)\left.\right|^{2}\right\rangle= & \frac{1}{2} \int_{-\infty}^{\lambda} m^{\prime}(\mu) d \mu \\
& \times\left|\int_{\mathbb{R}^{d}} \varphi^{*}(p)\left(\pi m^{\prime}(\mu)\right)^{-\frac{1}{2}} \ell(\mu, p) e^{t(\mu, p)+2 \pi i(n, p)} d p\right|^{2} .
\end{aligned}
$$

This relation together with (3.22) yield

$$
\left\langle\left(\varphi \mathscr{I}_{p}(\lambda) \varphi\right)\right\rangle=\int_{-\infty}^{\lambda} d \mu \int_{\mathbb{T}}\left|\varphi^{*}(p) \ell(\mu, p)\right|^{2} d p
$$

[in obtaining (3.24) we made use of (3.10) and (2.10)]. Equation (3.24), since $\varrho<\frac{1}{4}$, yields

$$
\left\langle\left(\varphi \mathscr{I}_{p}(d \lambda) \varphi\right)\right\rangle=\int_{B}|\varphi(p)|^{2}|\ell(\lambda, p)|^{2} d p d \lambda, \quad \varphi \in \mathscr{H}_{-}
$$

with $B=\left\{p^{2} \leq \varrho\right\}$. Now let us investigate the nondecreasing function $N(\lambda, \varphi)=$ $\langle(\varphi, E(\lambda) \varphi)\rangle, \varphi \in \mathscr{H}_{-}$, and the corresponding measure. Note that if $z \in L \cap$ $\{\operatorname{Im} \zeta>0\}$ then

$$
(\langle R(z)\rangle \varphi)(p)=\frac{\varphi(p)}{p^{2}-z}-\frac{\hat{v}(p)}{p^{2}-z} \frac{1}{w_{z}(p)+i}\left(\frac{\hat{v}(p) \varphi(p)}{p^{2}-z}\right)_{\mathbb{T}} .
$$

This equality follows from representations (1.14) and (3.7), since relations (2.5) allow to expand the operator $\left(1-e^{f_{0}(z)} \chi U\right)^{-1}$ in $\varkappa=e^{-2 \pi i \omega}$, and the result of applying the operation $\langle\ldots\rangle_{\mathbb{T}}$ will be the same as if we just set $x=0$. Equation (3.26) implies that

$$
\begin{aligned}
\operatorname{Im}(\varphi,\langle R(z)\rangle \varphi)= & \int_{B} d p \operatorname{Im}\left(p^{2}-z\right)^{-1}\left[|\varphi|^{2}-\operatorname{Re} v \varphi^{*}\left(\frac{v \varphi}{p^{2}-z}\right)_{\mathbb{T}}\left(w_{z}+i\right)^{-1}\right] \\
& \left.-\int_{B} d p \operatorname{Re}\left(p^{2}\right)-z\right)^{-1} \cdot \operatorname{Im} \hat{v} \varphi^{*}\left(\frac{\hat{v} \varphi}{p^{2}-z}\right)_{\mathbb{T}}\left(w_{z}+i\right)^{-1}
\end{aligned}
$$

Set $z=\lambda+i \varepsilon$ and tend $\varepsilon$ to zero. The first integral in (3.27) will tend to zero since the second factor of the integrand equals 0 at $z=\lambda+i 0$. Thus, after some algebra and taking account of the smoothness of $\varphi$ and Conditions V, we will deduce from (3.27)

$$
\operatorname{Im}(\varphi,\langle R(\lambda+i 0)\rangle \varphi)=\int_{\mathbb{T}}\left|\left(\frac{\hat{v} \varphi}{p^{2}-\lambda}\right)_{\mathbb{T}}\right|^{2}\left(w_{\lambda}^{2}+1\right)^{-1} d p .
$$

Having used the inequality $\varrho<\frac{1}{4}$ and equality (2.27), we shall get

$$
\operatorname{Im}(\varphi,\langle R(\lambda+i 0)\rangle \varphi)=\int|\ell(\lambda, p)|^{2}|\varphi(p)|^{2} d p, \quad \lambda \in \mathbb{R}, \quad \varphi \in S \cap \mathscr{H}_{-} .
$$


On the other hand, since $(\varphi,\langle R(z)\rangle \varphi)=\int_{-\infty}^{\infty}(\lambda-z)^{-1} d N(\lambda, \varphi)$, we shall get

$$
\pi^{-1} \operatorname{Im}(\varphi,\langle R(\lambda+i 0)\rangle \varphi)=\frac{\partial}{\partial \lambda} N(\lambda, \varphi)
$$

almost everywhere in the Lebesgue measure. Since, according to (3.29), the lefthand side of Eq. (3.30) exists and is bounded at $\lambda \in \mathbb{R}$, the measure $N(d \lambda, \varphi)$ is absolutely continuous [16] and

$$
N(d \lambda, \varphi)=\langle(\varphi, E(d \lambda) \varphi)\rangle=\int|\ell(\lambda, p)|^{2}|\varphi(p)|^{2} d p d \lambda, \quad \varphi \in S \cap \mathscr{H}_{-} .
$$

Denote an orthogonal projection onto $\mathscr{H}_{-}$by $\mathscr{J}$ and note that since $\tilde{u}_{n} \in \mathscr{H}_{-}$, than $\mathscr{I}(\lambda) \leq \mathscr{I}$. Besides, it is evident that

$$
\mathscr{I}_{p}(d \lambda) \leq E(d \lambda) \text { and } \mathscr{I} E(d \lambda)=E(d \lambda) \mathscr{I},
$$

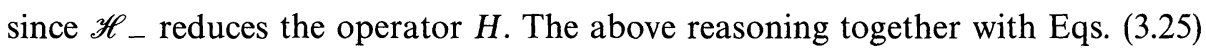
and (3.31) yield that $\left\langle\mathscr{I}_{p}(d \lambda)\right\rangle=\langle E(d \lambda) \mathscr{I}\rangle$ and, therefore, for almost all $\omega$ 's,

$$
\mathscr{I}_{p}(\omega, d \lambda)=E(\omega, d \lambda) \mathscr{I} .
$$

This equality means, in particular, that for almost all $\omega$ 's $\mathscr{I}_{p}(\omega, \infty)=\mathscr{I}$, i.e. the eigenfunctions $u_{\lambda_{n}}$ form a basis of the space $\mathscr{H}_{-}$. The lemma is proved.

Remark. In the course of writing this article the authors found that the proof of completeness in their article [6] has a gap. Nevertheless, the complete proof can be constructed out of assertions contained in [6]. To be exact, the completeness of the eigenvectors for almost all $\omega$ 's can be proved either as in Remarks in $[6$, p. 415] or as has been proved above. The completeness for all $\omega$ 's follows then from the observation that the projection $P$ onto the space generated by the eigenvector is strongly continuous with respect to $\omega$, i.e. $s-\lim _{\omega^{\prime} \rightarrow \omega} P\left(\omega^{\prime}\right)=P(\omega)$. This assertion immediately follows from representation (3.59) in [6].

Lemmas 3.3 to 3.5 imply Theorem 1.3 .

Lemma 3.6. Let $\varrho>\frac{1}{4}$ and $\mathscr{M}_{1}=\left(-\frac{1}{2}, m(\delta)\right)$ with the function $m(\lambda)$ and the number $\delta$ defined in Lemma 2.1.

Then

(a) the set of eigenvalues of the operator $H$ which lie in the interval $(-\infty, \delta)$ coincides with the set of solutions of Eq. (1.28) belonging to the above interval, exactly one solution corresponding to each $n$ such that

$$
(\alpha, n)+\omega \in \mathscr{M}_{1}(\bmod 1) ;
$$

(b) for each $n$ satisfying relation (3.33) there exists an eigenvalue $\lambda_{n} \in(-\infty, \delta)$ and an eigenfunction $u_{\lambda_{n}}$ of the operator $H$ such that Eq. (3.14) is valid and

$$
u_{\lambda_{n}}(p)=(-2 i) \frac{\hat{v}(p)}{p^{2}-\lambda_{n}}\left(w_{\lambda_{n}}(p)-i\right)^{-1} \exp \left\{t\left(\lambda_{n}, p\right)+2 \pi i(n, p)\right\}
$$

with $u_{\lambda_{n}} \in S \cap \mathscr{H}_{-}$;

(c) for almost all $\omega$ 's (in the Lebesgue measure) the spectrum of the operator $H$ in the $(-\infty, \delta)$ consists of the purely point component. 
The proof of the present lemma will be a literal repetition of the proof of the two preceding lemmas except the fact that here only part of the spectral axis coinciding with the $(-\infty, \delta)$ will be examined. The similarity follows from relations (2.12) and (2.15).

Lemmas 3.3 and 3.6 imply Theorem 1.2.

\section{Calculation of Integrated Density of States and Conductivity}

At first, examine the case $\varrho<\frac{1}{4}$. In calculating IDS of $H$ we will use the notation and relations from the proof of Lemma 3.5. Equations (1.6), (3.32) and (1.5) imply that

$$
\begin{aligned}
N(\lambda) & =\left\langle\operatorname{Sp} \hat{\chi}_{\mathbb{T}}(E(\lambda) \mathscr{I}+E(\lambda)(I-J)) \hat{\chi}_{\mathbb{T}}\right\rangle \\
& =\operatorname{Sp} \hat{\chi}_{\mathbb{T}}\left\langle\mathscr{I}_{p}(\lambda)\right\rangle \hat{\chi}_{\mathbb{T}}+\operatorname{Sp} \hat{\chi}_{\mathbb{T}} E_{0}(\lambda) \chi_{\varrho} \hat{\chi}_{\mathbb{T}},
\end{aligned}
$$

where $\chi_{p}$ is a characteristic function of the $[\varrho, \infty)$ and the operator of multiplying by the function $\chi_{p}\left(p^{2}\right)$ as well. The operators $\left\langle\mathscr{I}_{p}(\lambda)\right\rangle$ and $E_{0}(\lambda)$ are the operators of multiplying by the following functions of $p$ :

$$
\int_{-\infty}^{\lambda} d \mu|\ell(\mu, p)|^{2}, \quad \chi_{\varrho}\left(p^{2}\right) \chi\left(\lambda-p^{2}\right) .
$$

Note that the following is valid.

Lemma 4.1. Let $h(p), p \in \mathbb{R}^{d}$, be a nonnegative summable function, with $h$ being the operator of multiplication by this function. Then

$$
\operatorname{Sp} \hat{\chi}_{\mathbb{T}} h \hat{\chi}_{\mathbb{T}}=\int h(p) d p .
$$

Proof. Indeed, $\operatorname{Sp} \hat{\chi}_{\mathbb{T}} h \hat{\chi}_{\mathbb{T}}=\operatorname{Sp} \chi_{\mathbb{T}} \hat{h} \chi_{\mathbb{T}}$, the right-hand side being a nonnegative operator in $L_{2}(\mathbb{T})$ with the continuous kernel $\hat{h}(x-y), x, y \in \mathbb{T}$. But, according to [17], $\operatorname{Sp} \chi_{\mathbb{T}} \hat{h} \chi_{\mathbb{T}}=\left.\int_{\mathbb{T}} \hat{h}(x-y)\right|_{x=y} d x=\hat{h}(0)=\int h(p) d p$.

Combining (4.1), (4.2) and Lemma 4.1, we obtain

$$
N(\lambda)=\int_{-\infty}^{\lambda} d \mu \int|\ell(\mu, p)|^{2} d p+\int_{\varrho \leq p^{2} \leq \lambda} d p .
$$

From this equality together with Eqs. (2.26) to (2.28) we easily obtain formulas (1.7) to (1.9) which completes the proof of Theorem 1.4. Theorem 1.5 is proved in a similar way.

Before the proof of Theorem 1.6 we will give a definition of conductivity. To avoid cumbersome formulas we will consider a one-dimensional case $(d=1)$; it is clear that the results obtained can be generalized to the multi-dimensional case 
without essential changes in the reasoning. Let $z_{1}=\lambda+i \varepsilon, z_{2}=\lambda+v+i \varepsilon, \varepsilon>0$, $v>0$, while $R_{j}(\omega)=R\left(z_{j}, \omega\right), j=1,2$. Set

$$
\sigma^{(\varepsilon)}(v, \lambda)=-v^{-1} \int_{\lambda}^{\lambda+v}\left(1,\left\langle p\left(R_{1}-R_{1}^{*}\right) p\left(R_{2}-R_{2}^{*}\right)\right\rangle \mathbb{1}\right) d \lambda^{\prime},
$$

where $p$ is the operator of multiplication by the momentum $p$ and $\mathbb{1}$ is the function identically equal to 1 . The conductivity corresponding to the external field frequency $v$, to the Fermi energy $\lambda$ and to the zero temperature is given by the expression

$$
\sigma(v, \lambda)=\lim _{\varrho \downarrow 0} \sigma^{(\varepsilon)}(\varphi, \lambda) .
$$

This definition is based on the Kubo formula [8] derived in the framework of the linear response theory with an additional "averaging" over the energy interval of length $v$ in the vicinity of the Fermi energy. The factor including the electron charge, the Planck constant and the number $\pi$ is omitted from the formula.

Reducibility of the operator $H$ pointed out in Theorem 1.3 obviously implies a representation of the type given in Theorem 1.6 for $\sigma^{(\varepsilon)}$ and, hence, a similar representation for the conductivity $\sigma$, with

$$
\begin{gathered}
\sigma_{p}^{(\varepsilon)}(v, \lambda) \mid=-v^{-1} \int_{\lambda}^{\lambda+v}\left(\chi_{\varrho},\left\langle p\left(R_{1}-R_{1}^{*}\right) p\left(R_{2}-R_{2}^{*}\right)\right\rangle \chi_{p}\right) d \lambda^{\prime}, \\
\sigma_{p}(v, \lambda)=\lim _{\varrho \downarrow 0} \sigma_{p}^{(\varepsilon)}(v, \lambda) .
\end{gathered}
$$

Thus, one must get here an appropriate asymptotic estimate for the quantity $\sigma_{p}$ as $v \downarrow 0$. To this end, we will use the approach developed in [6]. Let $\eta=\eta(p)=e^{2 \pi i p}$. We will denote by $\eta$ the operator of multiplication by the function $\eta(p)$ in $L_{2}(\mathbb{T})$. Set $S^{1}=\{\zeta \in \mathbb{C}:|\zeta|=1\}$ and consider the set $\mathscr{F}$ of infinitely differentiable functions $a(\eta, \zeta)$ of the variables $\eta, \zeta \in S^{1}$. It is well-known that the function $a$ belongs to $\mathscr{F}$ if and only if it is expanded in the Fourier series,

$$
\begin{gathered}
a(\eta, \zeta)=\sum_{m, k \in \mathbb{Z}} \hat{a}_{m, k} \eta^{m} \zeta^{k}, \\
\hat{a}_{m, k}=(2 \pi i)^{-2} \int_{S^{1} \times S^{1}} a(\eta, \zeta) \eta^{-(m+1)} \zeta^{-(k+1)} d \eta d \zeta,
\end{gathered}
$$

and when its coefficients $\hat{a}_{m, k}$ decrease when $|m|+|k| \rightarrow \infty$ faster than any negative power of $|m|+|k|+1$. Each function $a \in \mathscr{F}$ can be put to one-to-one correspondence with the operator $a(\eta, U)$ in $L_{2}(\mathbb{T})$ defined by the equality

$$
a(\eta, U)=\sum_{m, k} \hat{a}_{m, k} \eta^{m} U^{k}
$$

Lemma 4.2. A set of operators of the form given by (4.10), when a runs through $\mathscr{F}$ forms the algebra $\mathscr{A}$. Here

$$
U \eta^{m}=\gamma^{m} \eta^{m} U, \quad \gamma=e^{-2 \pi i \alpha}
$$


and if to denote $\langle A(\omega)\rangle_{0}=\left(\eta^{0},\langle A\rangle \eta^{0}\right)$, then

$$
\left\langle a_{1} \ldots a_{s}\right\rangle_{0}=\left\langle a_{2} \ldots a_{s} a_{1}\right\rangle_{0},\left\langle a^{*}\right\rangle_{0}=\langle a\rangle_{0}^{*}, \quad a_{j} \in \mathscr{A} .
$$

The proof of the assertions of this lemma coincides, to within notations, with the proof of the similar assertions in [6]. It should be noted that the algebra considered in [6] differs from the algebra described here only by considering the functions with exponentially decreasing Fourier coefficients (i.e. analytical in an annulus) in the former case, while here the coefficients are assumed to be decreasing faster than any negative power. Beside the algebra $\mathscr{A}$ we will consider its extension $\tilde{\mathscr{A}}$. Let $\mathscr{A}_{0}$ be an algebra of operators of multiplication by bounded complex-values functions in $L_{2}(\mathbb{T})$. Then the set of finite sums of all possible finite products of operators from $\mathscr{A}$ and $\mathscr{A}_{0}$ obviously form an algebra which we will denote by $\tilde{\mathscr{A}}$.

Lemma 4.3. If $h$ is an operator of multiplication by the function $h(p) \in L_{2}(\mathbb{T}$, then

$$
U h=h_{\alpha} U, \quad h_{\alpha}(p)=h(p-\alpha) .
$$

Besides, if $a_{1}, \ldots, a_{s} \in \tilde{\mathscr{A}}$ then the relations (4.12) are valid.

Proof. Relation (4.13) immediately follows from (1.19); it is easy to establish (4.12) with the aid of (4.13).

Returning to the calculation of the integrand in (4.6) and using Lemma 4.3, we get

$$
\begin{aligned}
\left(\chi_{\varrho}\right. & \left.,\left\langle p\left(R_{1}-R_{1}^{*}\right) p\left(R_{2}-R_{2}^{*}\right)\right\rangle \chi_{\varrho}\right) \\
& =\left(\eta^{0},\left\langle\chi_{\varrho} p\left(R_{1}-R_{1}^{*}\right) p\left(R_{2}-R_{2}^{*}\right) \chi_{\varrho}\right\rangle \eta^{0}\right) \\
& =2 \operatorname{Re}\left\{\left\langle\chi_{\varrho} p R_{1} p R_{2} \chi_{\varrho}\right\rangle_{0}-\left\langle\chi_{\varrho} p R_{1} p R_{2}^{*} \chi_{\varrho}\right\rangle_{0}\right\}
\end{aligned}
$$

To calculate the latter expression, we shall need the following representations for the resolvent:

$$
\begin{gathered}
R(z)=\left(p^{2}-z\right)^{-1}-r, \\
r=\frac{\hat{v}}{p^{2}-z}(I-x U) e^{t}\left(I-x e^{f_{0}} U\right)^{-1} e^{-t}(w+i)^{-1} \frac{\hat{v}}{p^{2}-z}, \\
r=\frac{\hat{v}}{p^{2}-z} e^{t}\left(I-x e^{f_{0}} c^{-1} U\right)\left(I-x e^{f_{0}} U\right)^{-1} e^{-t}(w+i)^{-1} \frac{\hat{v}}{p^{2}-z},
\end{gathered}
$$

where, for shortness, the arguments $p$ and $z$ of the functions $\hat{v}, t, c, w, f_{0}$ are omitted. Formulas (4.15) and (4.16) follow from equalities (1.14) and (3.5), while formula (4.17) follows from (4.16) and (2.9). In the manner used in deriving equality (3.26) we can get

$$
\begin{gathered}
\langle r\rangle=\left(\frac{\hat{v}}{p^{2}-z}\right)^{2}(w+i)^{-1}=\frac{1}{p^{2}-z} \frac{w}{w+i}, \\
\langle R(z)\rangle=\frac{1}{p^{2}-z} \cdot \frac{1 i}{w+i}, \\
\left\langle p R_{1} p R_{2}\right\rangle=p\left\langle R_{1}\right\rangle p\left\langle R_{2}\right\rangle=-\frac{p^{2}}{\left(p^{2}-z_{1}\right)\left(p^{2}-z_{2}\right)} \cdot \frac{1}{\left(w_{1}+i\right)\left(w_{2}+i\right)} .
\end{gathered}
$$


Here and further on the subscripts 1 and 2 at the functions $w, t, c$ and $f_{0}$ mean that the arguments are $z_{1}$ and $z_{2}$, respectively. Relations (4.15), (4.18), and (4.19) imply

$$
\begin{aligned}
\left\langle p R_{1} p R_{2}^{*}\right\rangle= & \frac{p^{2}}{\left(p^{2}-z_{1}\right)\left(p^{2}-z_{2}\right)}\left(\frac{i}{w_{2}+i}\right)^{*} \\
& -\frac{p^{2}}{\left.p^{2}-z_{1}\right)} \cdot \frac{w_{1}}{w_{1}+i} \cdot \frac{1}{p^{2}-z_{2}^{*}}+\left\langle p r_{1} p r_{2}^{*}\right\rangle
\end{aligned}
$$

To calculate the right-hand side of Eq. (4.21) we will introduce the auxiliary functions

$$
\begin{aligned}
b_{z}^{ \pm}(p) & =\frac{1}{\left(p^{2}-z\right)\left(w_{z} \mp i\right)}, B_{z}^{ \pm}(p)=\hat{v}(p) b_{z}^{ \pm}(p), \\
V^{ \pm}(p) & =V_{1,2}^{ \pm}(p)=p e^{ \pm t_{1}(p)} B_{1}^{ \pm}(p) e^{ \pm t_{2}^{*}(p)}\left(B_{2}^{ \pm}\right)^{*}(p)
\end{aligned}
$$

Lemma 4.4. Let $Y_{\delta}=\left\{\zeta \in \mathbb{C}:|\operatorname{Re} \zeta-\varrho|>\delta,|\operatorname{Im} \zeta|<\frac{1}{2} a(\delta)\right\}$, where $a$ is the function mentioned in Conditions $V$. Then for a small enough $\delta$ the functions $b_{z}^{ \pm}(p)$, $B_{z}^{ \pm}(p), V_{z_{1}, z_{2}}^{ \pm}(p)$ are infinitely differrentiable at $p \in \mathbb{T}, z, z_{1}, z_{2} \in Y_{\delta}$. Besides

$$
w \chi_{\varrho}=w, \quad B^{ \pm} \chi_{\varrho}=B^{ \pm}, \quad V^{ \pm} \chi_{\varrho}=V^{ \pm} .
$$

Proof. Identities (4.24) obviously follow from Conditions $V$, while smoothness of the functions $b^{ \pm}, B^{ \pm}$and $V^{ \pm}$immediately follow from Definitions (1.15), (4.22), (4.23) and Lemma 2.2.

In particular, Lemma 4.4 implies that if the Fourier expansion of the function $V^{ \pm}$has the form

$$
V^{ \pm}=\sum_{m \in \mathbb{Z}} \beta_{m}^{ \pm} \eta^{m}
$$

and $\min \{|\lambda-\varrho|,|\lambda+\varphi-\varrho|\}=\delta>0$, then for $\varepsilon<a(\delta) / 2$ and any natural $n$

$$
\left|\beta_{m}^{ \pm}\right| \leq C(n, \delta)(|m|+1)^{-n},
$$

where $C(n, \delta)$ is a quantity depending only on $n$ and $\delta$. Equalities (4.17), (4.23), (4.25) and (4.11) imply

$$
\begin{aligned}
& \left\langle p r_{1} p r_{2}^{*}\right\rangle=\left\langle p \frac{\hat{v}}{p^{2}-z_{1}} e^{t_{1}}\left(I-x e^{f_{0,1}} c_{1}^{-1} U\right)\left(I-x e^{f_{0,1}} U\right)^{-1}\right. \\
& \left.\times V^{-}\left(I-\varkappa^{-1} e^{f_{0,2}^{*}} U^{-1}\right)^{-1}\left(I-\varkappa^{-1} e^{f_{0,2}^{*}} U^{-1}\left(c_{2}^{-1}\right)^{*}\right) e^{t_{2}^{*}} \frac{\hat{v}}{p^{2}-z_{2}^{*}}\right\rangle \\
& =\sum_{m} \beta_{m}^{-}\left\langle p \frac{\hat{v}}{p^{2}-z_{1}} e^{t_{1}} \eta^{m}\left(I-x e^{f_{0,1}} \gamma^{m} e_{1}^{-1} U\right)\left(I-\varkappa e^{f_{0,1}} \gamma^{m} U\right)^{-1}\right. \\
& \left.\times\left(I-x^{-1} e^{f_{0,2}^{*}} U^{-1}\right)\left(I-\chi^{-1} e^{f_{0,2}^{*}} U^{-1}\left(c_{2}^{-1}\right)^{*}\right) e^{t_{2}^{*}} \frac{\hat{v}}{p^{2}-z_{2}^{*}}\right\rangle
\end{aligned}
$$




$$
\begin{aligned}
= & \sum_{m} \beta_{m}^{-} p \frac{\hat{v}}{p^{2}-z_{1}} e^{t_{1}} \eta^{m}\left[\left(c_{2}^{*}\right)^{-1}+\left(1-e^{\left.f_{0,1}+f_{0,2}^{*} c_{1}^{-1}\right)}\right.\right. \\
& \left.\times\left(1-e^{f_{0,1}+f_{0,2}^{*} \gamma^{m}}\right)^{-1}\left(1-\left(c_{2}^{*}\right)^{-1}\right)\right] e^{t_{2}^{*}} \frac{\hat{v}}{p^{2}-z_{2}^{*}} .
\end{aligned}
$$

The latter of the equalities obtained can be derived as was done in [6] in the similar case. If we add and subtract 1 at $c_{1}^{-1}$ in the latter equality, then, by using the identity $1-c^{-1}=-2 i(w-i)^{-1}$, we get

$$
\begin{aligned}
\left\langle p r_{1} p r_{2}^{*}\right\rangle= & \sum_{m} \beta_{m}^{-} p \frac{\hat{v}}{p^{2}-z_{1}} e^{t_{1}} \eta^{m} \\
& {\left[1+\frac{4}{\left(w_{1}-i\right)\left(w_{2}-i\right)} \cdot \frac{e^{f_{0,1}+f_{0,2}^{*} \gamma^{m}}}{1-e^{f_{0,1}+f_{0,2}^{*} \gamma^{m}}}\right] e^{t_{2}^{*}} \frac{\hat{v}}{p^{2}-z_{2}^{*}} . }
\end{aligned}
$$

Combining the identity $x(1-x)=\frac{1}{2}\left[(1+x)(1-x)^{-1}-1\right]$ with Eqs. (4.22) and (4.23) we, after simple algebra, get from the latter equality that

$$
\begin{aligned}
\left\langle p r_{1} p r_{2}^{*}\right\rangle= & p^{2} \frac{w_{1}}{\left(p^{2}-z_{1}\right)\left(w_{1}+i\right)} \cdot \frac{w_{2}^{*}}{\left(p^{2}-z_{2}\right)\left(w_{2}+i\right)} \\
& -2 p^{2} B_{1}^{+} B_{1}^{-}\left(B_{2}^{+} B_{2}^{-}\right)^{*}+\Sigma \\
\Sigma & =2 \sum_{m} \beta_{m}^{-} V^{+} \eta^{m} \frac{1+e^{f_{0,1}+f_{0,2}^{*} \gamma^{m}}}{1-e^{f_{0,1}+f_{0,2}^{*} \gamma^{m}}} .
\end{aligned}
$$

Thus, Eqs. (4.20) to (4.23), (4.28), and (4.29) will yield, after some elementary transformations,

$$
\begin{aligned}
& \left\langle p R_{1} p R_{2}^{*}\right\rangle=-p^{2} b_{1}^{-} b_{2}^{-}, \\
& \left\langle p R_{1} p R_{2}^{*}\right\rangle=p^{2}\left[b_{1}^{-} b_{2}^{-*}-2 B_{1}^{-} B_{1}^{+}\left(B_{2}^{-} B_{2}^{+}\right)^{*}\right]+\Sigma,
\end{aligned}
$$

and further, using Lemma 4.4 and identity (4.24),

$$
\begin{aligned}
& \left.\left\langle\chi_{\varrho} p\left(R_{1}-R_{1}^{*}\right) p\left(R_{2}-R_{2}^{*}\right) \chi_{\varrho}\right\rangle_{0}\right|_{\varepsilon=+0} \\
& \quad=-\left.2 \operatorname{Re} \Sigma_{0}\right|_{\varepsilon=+0}-2 \operatorname{Re}\left\{\left(\chi_{\varrho},\left.p^{2}\left[b_{1}^{-}\left(b_{2}^{-}+b_{2}^{-*}\right)-2 B_{1}^{-} B_{1}^{+}\left(B_{2}^{-} B_{2}^{+}\right)^{*}\right]\right|_{\varepsilon=+0} \chi_{\varrho}\right\}\right. \\
& \quad=-\left.2 \operatorname{Re} \Sigma_{0}\right|_{\varepsilon=+0},
\end{aligned}
$$

where

$$
\Sigma_{0}=\left(\eta^{0}, \Sigma \eta^{0}\right)=\sum_{m} \beta_{m}^{-} \beta_{-m}^{+} \frac{1+e^{f_{0,1}+f_{0,2}^{*} \gamma^{m}}}{1-e^{f_{0,1}+f_{0,2}^{*} \gamma^{m}}} .
$$

Bringing together relations (4.6), (4.7), (4.14), (4.30) and (4.31), we get for $\lambda \neq \varrho$ and $v \leq \frac{1}{2}|\lambda-\varrho|$,

$$
\sigma_{p}(v, \lambda)=2 v^{-1} \int_{\lambda}^{\lambda+v} \operatorname{Re} \Sigma_{0}\left(\lambda^{\prime}\right) d \lambda^{\prime} .
$$

Now we have only to note that if to use relations (4.26), (4.31) and (4.32) and to repeat the argumentation concerning similar expressions from [6], then we get that $\sigma_{p}(v, \lambda)$ decrease for $v \downarrow 0$ faster that any power of $v$. Theorem 1.6 is proved. 
Acknowledgement. L. P. would like to thank Prof. W. Thirring for the encouraging discussions on the status of nonlocal potentials in theoretical physics during IX-th IAMP Congress, Swansea, 1988.

\section{References}

1. Pastur, L.A.: Spectral properties of disordered systems in the one-body approximation. Commun. Math. Phys. 75, 179-196 (1980)

2. Pastur, L.A., Figotin, A.L.: Ergodic properties of the distribution of eigenvalues of certain classes of random self-adjoint operators. Selecta. Math. Sov. 3, 69-96 (1983)

3. Figotin, A.L.: Essential selfadjointness and ergodic properties of the Schrödinger operator with the random potential. Dokl. Ak. Nauk Ukr SSR, N 8, 18-20 (1980) (in Russian)

4. Fishman, S., Grempel, D.R., Prange, R.E.: Localization in an incommensurate potential: an exactly soluble model. Phys. Rev. Lett. 49, 833-836 (1982)

5. Simon, B.: Almost periodic Schrödinger operators. IV. The Maryland model. Ann. Phys. (USA) 159, 157-183 (1985)

6. Figotin, A.L., Pastur, L.A.: An exactly soluble model of a multidimensional incommensurate system. Commun. Math. Phys. 95, 401-425 (1984)

7. Albeverio, S., Gesztesy, F., Hoegh-Krohn, R., Holden, H.: Solvable models in quantum mechanics. Berlin, Heidelberg, New York: Springer 1988

8. Lifshitz, I.M., Gredeskul, S.A., Pastur, L.A.: Introduction to the theory of disordered systems. New York: Wiley 1988

9. Lifshitz, I.M.: On the structure of the energy spectrum and quantum states of disordered condenses systems. Sov. Phys. Usp. 7, 543-581 (1965); The theory of fluctuation levels in disordered systems. Zh. Eks. Teor. Fiz. 53, 743-758 (1967) (in Russian)

10. Martinelli, F., Scoppolla, E.: Introduction to the mathematical theory of Anderson localization. Riv. Nuovo Cimento 10, 2-90 (1987)

11. Prange, R.E., Grempel, D.R., Fishman, S.: Long range resonance in Anderson insulators: finite frequency conductivity of random and incommensurate systems. Phys. Rev. Lett. 53, 1582-1585 (1985)

12. Lee, P.A., Ramakrishnan, T.V.: Disordered electronic systems. Rev. Mod. Phys. 57, 287-337 (1985)

13. Souillard, B., Devillard, P.: Polynomially decaying transmission for the nonlinear Schrödinger equation in a random potential. J. Stat. Phys. 43, 423-439 (1986)

14. Fröhlich, J., Spencer, T., Wayne, E.: Localization in disordered nonlinear dynamical systems. J. Stat. Phys. 42, 247-271 (1987)

15. Bellissard, J., Vittot, P.: Preprint, Marseille, 1987

16. Reed, M., Simon, B.: Methods of modern mathematical physics. IV. Analysis of operators. New York: Academic Press 1978

17. Berezanski, Yu.M.: Expansions in eigenfunctions of selfadjoint operators. Transl. Math. Monographs AMS 17, 1968

18. Albanese, C., Fröhlich, J.: Periodic solutions of some infinite-dimensional hamiltonian systems associated with non-linear partial differential equations. I. Commun. Math. Phys. 116, 539-572 (1988)

19. Dinaburg, E.I., Sinai, Ya.G.: The one dimensional Schrödinger equation with a quasiperiodic potential. Funct. Anal. Appl. 9, 279-289 (1975)

20. Sinai, Ya.G.: Anderson localization for one-dimensional difference Schrödinger operator with quasiperiodic potential. J. Stat. Phys. 46, 861-909 (1987)

21. Chulaevsky, V., Delyon, F.: Purely absolutely continuous spectrum for almost Mathieu operator, J. Stat. Phys. 55, 1279-1285 (1989)

22. Eliasson, L.H.: Floquet solutions for the 1-dimensional quasiperiodic Schrödinger equation. ETH Preprint

Communicated by Ya. G. Sinai 